九州大学学術情報リポジトリ

Kyushu University Institutional Repository

\title{
ON THE DYNAMIC PROBABILISTIC INVENTORY PROBLEMS WITH PIECEWISE COST FUNCTIONS WHICH MAY NOT BE PIECEWISE SMOOTH
}

Sakaguchi, Michinori

Faculty of Economic Sciences, Hiroshima Shudo University

Kodama, Masanor i

Faculty of Economic Sciences, Hiroshima Shudo University

https://doi.org/10.5109/13510

出版情報: Bulletin of informatics and cybernetics. 34 (1), pp.75-90，2002-10. Research Association of Statistical Sciences

バージョン :

権利関係 : 


\title{
ON THE DYNAMIC PROBABILISTIC INVENTORY PROBLEMS WITH PIECEWISE COST FUNCTIONS WHICH MAY NOT BE PIECEWISE SMOOTH
}

\author{
By \\ Michinori Sakaguchi* and Masanori Kodama ${ }^{\dagger}$
}

\begin{abstract}
A mathematical model of the probabilistic inventory problems with piecewise cost functions which may not be piecewise smooth is presented and various properties in this model are studied. Also some sufficient conditions on cost functions are found to ensure simple treatment on an optimal policy.
\end{abstract}

Key Words and Phrases: Dynamic inventory problem, Dynamic programming, Piecewise cost function.

\section{Introduction}

We consider the optimal policy of a probabilistic inventory problem with a piecewise cost function. The decision criterion is the maximization of expected profit or the minimization of expected costs which include the ordering, holding, and shortage costs. A typical example in single period problem is as follows. Let $x$ be the amount on hand before an order is placed and let $\phi(b)$ be the probability density function of demand $B$. Let $h$ and $p$ be the holding and shortage costs per unit per period. Further, let $c$ be the purchasing cost per unit and let $z$ be the amount on hand in initial period after an order is received, which means that the initial regular order is $z-x$. If we assume no setup cost is occurred, the expected cost for the period is given by

$$
E\{C(B, z)\}=c(z-x)+h E\{\text { holding cost }\}+p E\{\text { shortage cost }\} .
$$

We define the function $H(z)$ by the equation $E\{C(B, z)\}=-c x+H(z)$ and let $f_{1}(x)$ be the minimal expectation of the total cost. Then we may write

$$
f_{1}(x)=\min _{z \geq x}\{-c x+H(z)\}
$$

In one period problem the maximization (Kabak, 1984, Sorai, Arizono and Ohta, 1986) and the minimization (Kodama and Kitahara, 1983, Kodama, 1986) of the expected function in inventory models are considered and the special inventory problems are solved in Kodama (1990a) and Kodama (1991a). Moreover, a mathematical generalization of

\footnotetext{
* Faculty of Economic Sciences, Hiroshima Shudo University 1-1 Ohtsukahigasi-1-chome, Asaminamiku, Hiroshima-si, 731-3195, Japan. tel +81-82-830-1237 sakaguti@shudo-u.ac.jp

$\dagger$ Faculty of Economic Sciences, Hiroshima Shudo University 1-1 Ohtsukahigasi-1-chome, Asaminamiku, Hiroshima-si, 731-3195, Japan. tel +81-82-830-1942 kodama@shudo-u.ac.jp
} 
the cost functions in the inventory problem is defined and analyzed in Kodama (1990b), Kodama (1992) and Kodama (1996).

In multiperiod models we suppose that $\phi(b)$ remains unchanged from period to period and demands in each period are independent. We should take in the discounted value of money in this case. That is, if $\alpha(<1)$ is the discount factor per period and $f_{n}(x)$ is the discount expected loss for $n$-period inventory model when an optimal policy is used at each purchaing opportunity, then

$$
f_{n}(x)=\min _{z \geq x}\left\{-c x+H(z)+\alpha \int_{0}^{\infty} f_{k-1}(z-b) \phi(b) d b\right\}
$$

Various properties of the optimal policy in multi-stage model are studied in Kodama (1991b) and Kodama (1993). In recent papers (Kodama, 1998a and Kodama, 1998b), we have attempted to express the optimal function by closed forms with known cost functions in the multi-stage model. Furthermore, some sufficient conditions on cost functions are found to ensure simple treatments on an optimal policy and specializations of cost functions are made and many examples are analyzed. In Kodama and Sakaguchi (2001c) we studied inventory models with a piecewise cost function that was piecewise smooth and discussed the properties of the optimal policy. We define functions $F_{k}(z)$ by the equations

$$
F_{k-1}(z)=H(z)+\alpha \int_{0}^{\infty} f_{k-1}(z-b) \phi(b) d b, \quad f_{0}(\cdot)=0, \quad k=1,2, \cdots, N
$$

and let

$$
\bar{x}_{n}=\inf \left\{z \mid F_{n-1}^{\prime}(z) \geq 0\right\} .
$$

Then the optimal policy in the probabilistic dynamic inventory problem was given as follows:

$$
\text { If } x<\bar{x}_{n} \text {, then order }\left(\bar{x}_{n}-x\right) \text {, otherwise do not order. }
$$

In this paper we give a mathematical model of dynamic inventory problems with a piecewise cost function which may not be piecewise smooth. That is to say, we assume that the function $H(z)$ is a piecewise function which may not be piecewise smooth. In this case the fundamental properties of the functions $F_{k}(z)$ are given in Theorem 2.6 and we would set

$$
\bar{x}_{n}=\inf \left\{z \mid F_{(n-1)+}^{\prime}(z) \geq 0\right\} .
$$

We assume that $H_{i}(z) \quad(1 \leq i \leq m+1)$ has a continuous second derivative and is a convex function on $\left[R_{i-1}, R_{i}\right]$ such that $H(z)=H_{i}(z)$ for $z \in\left(R_{i-1}, R_{i}\right]$. It is effective to decide the sign of $F_{(n-1)+}^{\prime}\left(R_{i}\right)$ in order to get $\bar{x}_{n}$ and we show some sufficient conditions for this in terms of the discount factor $\alpha$ and $H\left(R_{i}\right)$ in Corollary to Theorem 3.1. Further we discuss the method to obtain $\vec{x}_{n}$ in some cases expressing the equation to solve by the known functions $H_{i}(z)$. 


\section{A mathematical model}

Let $c, \alpha$ be real numbers with $0<c, 0<\alpha<1$. Let $R_{1}, \ldots, R_{m}$ be a sequence of real numbers such that $R_{1}<\cdots<R_{m}$. Let $H_{i}(z) \quad(1 \leq i \leq m+1)$ be real valued-functions defined on $\left[R_{i-1}, R_{i}\right]$, where we respectively abbreviate $\left(-\infty, R_{1}\right]$ to $\left[R_{0}, R_{1}\right]$ and $\left[R_{m}, \infty\right)$ to $\left[R_{m}, R_{m+1}\right]$. Throughout this paper we assume that for all $i$ with $1 \leq i \leq m+1, H_{i}(z)$ has a continuous second derivative on $\left[R_{i-1}, R_{i}\right]$, and it is a convex function on $\left[R_{i-1}, R_{i}\right]$ which means by the condition that $H_{i}^{\prime \prime}(z) \geq 0$ on $\left(R_{i-1}, R_{i}\right)$. We also assume that

$$
\begin{gathered}
H_{i}\left(R_{i}\right)=H_{i+1}\left(R_{i}\right) \quad \text { and } H_{i}^{\prime}\left(R_{i}\right) \leq H_{i+1}^{\prime}\left(R_{i}\right) \text { for all } i \quad 1 \leq i \leq m \\
\lim _{z \rightarrow-\infty} H_{1}^{\prime}(z)<0, \quad \lim _{z \rightarrow \infty} H_{m+1}^{\prime}(z)>c .
\end{gathered}
$$

Now set

$$
H(z)=H_{i}(z) \quad \text { for } \quad z \in\left(R_{i-1}, R_{i}\right] .
$$

Then $H(z)$ has a derivative at every point in the set $\boldsymbol{R}$ of all real numbers except $z=R_{i} \quad 1 \leq i \leq m$. We know that $H_{-}^{\prime}\left(R_{i}\right)$ and $H_{+}^{\prime}\left(R_{i}\right)$ exist, and that the inequality

$$
H_{-}^{\prime}\left(R_{i}\right) \leq H_{+}^{\prime}\left(R_{i}\right)
$$

holds for all $i$ and we see that $H_{+}^{\prime}(z)$ is non-decreasing on $(-\infty, \infty)$.

Let $\phi(b)$ be the density function of a real random variable $B$ and we assume that $\phi(b)$ is a piecewise continuous function with $\phi(b)=0$ for $b<0$. For a given real number $x$ and $z$ we define functions $f_{k}(x), F_{k}(x)(k=1, \cdots, N)$ as follows.

$$
\begin{gathered}
f_{1}(x)=\min _{z \geq x}\{-c x+H(z)\}, \\
f_{k}(x)=\min _{z \geq x}\left\{-c x+H(z)+\alpha \int_{0}^{\infty} f_{k-1}(z-b) \phi(b) d b\right\}, \quad k=2,3, \cdots, N, \\
F_{k-1}(z)=H(z)+\alpha \int_{0}^{\infty} f_{k-1}(z-b) \phi(b) d b, \quad f_{0}(\cdot)=0, \quad k=1,2, \cdots, N .
\end{gathered}
$$

By $(2.1),(2.2),(2.3)$ and the fact that $H_{i}(z)$ is a convex function on $\left[R_{i-1}, R_{i}\right]$ for all $i(1 \leq i \leq m+1)$, there exists $\bar{x}_{1}$ such that

$$
\bar{x}_{1}=\inf \left\{z \mid H_{+}^{\prime}(z) \geq 0\right\} .
$$

Note that if $z \neq R_{i}$, then we have $H_{+}^{\prime}(z)=H^{\prime}(z)$.

LEMMA 2.1. We have

$$
f_{1}(x)= \begin{cases}-c x+H\left(\bar{x}_{1}\right), & \text { if } x \leq \bar{x}_{1}, \\ -c x+H(x), & \text { if } x \geq \bar{x}_{1} .\end{cases}
$$

In particular $f_{1}(x)$ is continuous on $\boldsymbol{R}$.

Proof. By the assumption (2.1), we know that $H_{+}^{\prime}(z)$ is a non-decreasing function on $(-\infty, \infty)$. By $(2.8)$ we see that $H\left(\bar{x}_{1}\right)$ is the minimum of $H(z)$ on the interval $(-\infty, \infty)$. It thus follows that

$$
\min _{z \geq x} H(z)= \begin{cases}H\left(\bar{x}_{1}\right), & \text { if } x \leq \bar{x}_{1}, \\ H(x), & \text { if } x>\bar{x}_{1}\end{cases}
$$


and hence we get the equation in this lemma.

Now we shall discuss some of the basic properties of the functions $f_{k}(x), F_{k}(x)(k=$ $1, \cdots, N)$. At first it will be shown that the function $F_{1}(z)$ has the similar properties to the function $H(z)$.

LEMMA 2.2. We have

$$
F_{1}(z)= \begin{cases}H(z)-\alpha c z+\alpha\left\{H\left(\bar{x}_{1}\right)+c E(B)\right\}, & \text { if } z \leq \bar{x}_{1}, \\
H(z)-\alpha c z+\alpha \int_{0}^{z-\bar{x}_{1}}\left\{\begin{array}{c}
\left\{H(z-b)-H\left(\bar{x}_{1}\right)\right\} \phi(b) d b \\
+\alpha\left\{H\left(\bar{x}_{1}\right)+c E(B)\right\},
\end{array}\right. & \text { if } z>\bar{x}_{1} .\end{cases}
$$

Furthermore $F_{1}(z)$ is continuous on $\boldsymbol{R}$.

Proof. Assume first that $z \leq \bar{x}_{1}$. Then it is shown that if $b \geq 0$, then $z-b \leq \bar{x}_{1}$ and hence $f_{1}(z-b)=-c(z-b)+H\left(\bar{x}_{1}\right)$ by Lemma 2.1 . We therefore obtain

$$
\begin{aligned}
F_{1}(z) & =H(z)+\alpha \int_{0}^{\infty}\left\{-c(z-b)+H\left(\bar{x}_{1}\right)\right\} \phi(b) d b \\
& =H(z)-\alpha c z+\alpha\left\{H\left(\bar{x}_{1}\right)+c E(B)\right\} .
\end{aligned}
$$

Next suppose that $z \geq \bar{x}_{1}$. Then $z-b \leq \bar{x}_{1}$ if and only if $z-\bar{x}_{1} \leq b$. Thus we have

$$
\begin{aligned}
F_{1}(z)= & H(z)+\alpha \int_{0}^{\infty} f_{1}(z-b) \phi(b) d b \\
= & H(z)+\alpha \int_{0}^{z-\bar{x}_{1}}\{-c(z-b)+H(z-b)\} \phi(b) d b \\
& +\alpha \int_{z-\bar{x}_{1}}^{\infty}\left\{-c(z-b)+H\left(\bar{x}_{1}\right)\right\} \phi(b) d b \\
= & H(z)-\alpha c z+\alpha \int_{0}^{z-\bar{x}_{1}}\left\{H(z-b)-H\left(\bar{x}_{1}\right)\right\} \phi(b) d b+\alpha\left\{H\left(\bar{x}_{1}\right)+c E(B)\right\},
\end{aligned}
$$

and we complete the proof because it is clear that $F_{1}(z)$ is continuous. given by

LeMmA 2.3. $\quad F_{1}(z)$ has a derivative on $\left[R_{i-1}, R_{i}\right]$ for all $i \quad(1 \leq i \leq m+1)$,

$$
F_{1}^{\prime}(z)= \begin{cases}H^{\prime}(z)-\alpha c, & \text { if } z \leq \bar{x}_{1} \text { and } z \neq R_{j}, \\ H^{\prime}(z)-\alpha c+\alpha \int_{0}^{z-\bar{x}_{1}} H^{\prime}(z-b) \phi(b) d b, & \text { if } z>\bar{x}_{1} \text { and } z \neq R_{j}\end{cases}
$$

where $j=1, \ldots, m$. We have

$$
F_{1+}^{\prime}\left(R_{i}\right)= \begin{cases}H_{+}^{\prime}\left(R_{i}\right)-\alpha c, & \text { if } R_{i} \leq \bar{x}_{1}, \\ H_{+}^{\prime}\left(R_{i}\right)-\alpha c+\alpha \int_{0}^{R_{i}-\bar{x}_{1}} H^{\prime}\left(R_{i}-b\right) \phi(b) d b, & \text { if } R_{i}>\bar{x}_{1}\end{cases}
$$

and

$$
F_{1-}^{\prime}\left(R_{i}\right)= \begin{cases}H_{-}^{\prime}\left(R_{i}\right)-\alpha c, & \text { if } R_{i} \leq \bar{x}_{1}, \\ H_{-}^{\prime}\left(R_{i}\right)-\alpha c+\alpha \int_{0}^{R_{i}-\bar{x}_{1}} H^{\prime}\left(R_{i}-b\right) \phi(b) d b, & \text { if } R_{i}>\bar{x}_{1},\end{cases}
$$

in particular we see $F_{1-}^{\prime}\left(R_{i}\right) \leq F_{1+}^{\prime}\left(R_{i}\right)$ for all $i(1 \leq i \leq m)$. 
Proof. Let $\bar{x}_{1}$ be an element in the interval $\left[R_{p-1}, R_{p}\right]$. If $z \leq \bar{x}_{1}$, our lemma is clear by Lemma 2.2. Therefore we may assume $\bar{x}_{1}<z$. It also follows from Lemma 2.2 that we may only study a derivative of the function

$$
\int_{0}^{z-\bar{x}_{1}}\left\{H(z-b)-H\left(\bar{x}_{1}\right)\right\} \phi(b) d b .
$$

For the sake of it we suppose that $z$ is on the interval $\left[R_{i-1}, R_{i}\right]$. Then we see $p \leq i$ and have a sequence

$$
R_{p-1}<\bar{x}_{1} \leq R_{p}<\cdots<R_{k-1}<R_{k}<\cdots<R_{i-1} \leq z \leq R_{i} .
$$

Put $g(z, b)=\left\{H(z-b)-H\left(\bar{x}_{1}\right)\right\} \phi(b)$ and let

$$
K=\left\{(z, b) \mid R_{i-1} \leq z \leq R_{i}, 0 \leq b \leq z-\bar{x}_{1}\right\}
$$

Then the function $H(z-b)$ of two real variables $z, b$ are continuous on the set $K$. Since $z-R_{k} \leq b \leq z-R_{k-1}$ if and only if $R_{k-1} \leq z-b \leq R_{k}$, we see that the function $H^{\prime}(z-b)$ of two real variables $z, b$ are continuous on the set $K-\cup_{k} L_{k}$, where $L_{k}=\left\{(z, b) \mid b=z-R_{k}\right\}(p \leq k \leq i-1)$. If $\phi(b)$ is continuous on [0, $\left.z-\bar{x}_{1}\right]$ and $R_{i-1}<z<R_{i}$, then

$$
\frac{d}{d z} \int_{0}^{z-\bar{x}_{1}} g(z, b) d b=\int_{0}^{z-\bar{x}_{1}} \frac{\partial g}{\partial z}(z, b) d b+g\left(z, z-\bar{x}_{1}\right)=\int_{0}^{z-\bar{x}_{1}} H^{\prime}(z-b) \phi(b) d b .
$$

Similarly we obtain that if $z=R_{i-1}$, then

$$
D_{+} \int_{0}^{R_{i-1}-\bar{x}_{1}} g\left(R_{i-1}, b\right) d b=\int_{0}^{R_{i-1}-\bar{x}_{1}} H^{\prime}\left(R_{i-1}-b\right) \phi(b) d b,
$$

and that if $z=R_{i}$, then

$$
D_{-} \int_{0}^{R_{i}-\bar{x}_{1}} g\left(R_{i}, b\right) d b=\int_{0}^{R_{i}-\bar{x}_{1}} H^{\prime}\left(R_{i}-b\right) \phi(b) d b .
$$

We have assumed that the function $\phi(b)$ is piecewise continuous, so suppose that $\phi(b)$ is discontinuous at $b=a_{j}(1 \leq j \leq l)$, where $a_{1}, a_{2}, \ldots, a_{l}$ is an increasing sequence with $a_{j} \in\left[0, z-\bar{x}_{1}\right]$.

At first suppose that $z \in\left(R_{i-1}, R_{i}\right)$. In this case there is a positive number $\delta$ such that the following conditions:

1. We have $R_{i-1} \leq z-\delta, z+\delta \leq R_{i}$.

2. If $z-\delta \leq w<z$, then $w-R_{k} \neq a_{j}$ for all $j, k$.

3. If $z<w \leq z+\delta$, then $w-R_{k} \neq a_{j}$ for all $j, k$.

Let

$$
\begin{aligned}
& K_{\delta-}=\left\{(w, b) \mid z-\delta \leq w \leq z, 0 \leq b \leq z-\bar{x}_{1}\right\} \\
& K_{\delta+}=\left\{(w, b) \mid z \leq w \leq z+\delta, 0 \leq b \leq z-\bar{x}_{1}\right\}
\end{aligned}
$$


Considering the fact that the function $H^{\prime}(z-b) \phi(b)$ of two real variables $z, b$ are continuous at every point in the set $K_{\delta}$ - which is not on any lines $b=z-R_{k}$ ( $p \leq$ $k \leq i-1)$ and $b=a_{j}(1 \leq j \leq l)$, we are able to calculate a left-hand derivative by the method on $K_{\delta-}$ used in (2.11). Similarly by the same method on $K_{\delta+}$ used in (2.10), we may have a right-hand derivative at $z$, and these are shown to be equal. This implies that (2.9) holds in this case. There are remained cases where $z=R_{i-1}$ or $z=R_{i}$, and the equations (2.10) and (2.11) can be shown similarly in these cases.

The inequality

$$
H_{-}^{\prime}\left(R_{i}\right) \leq H_{+}^{\prime}\left(R_{i}\right)
$$

leads us to the relations

$$
F_{1-}^{\prime}\left(R_{i}\right) \leq F_{1+}^{\prime}\left(R_{i}\right)
$$

for all $i(1 \leq i \leq m)$, therefore we complete our proof.

LEMMA 2.4. $\quad F_{1}(z)$ has a second continuous derivative on $\left[R_{i-1}, R_{i}\right]$ for all $i(1 \leq$ $i \leq m+1)$, given

$$
F_{1}^{\prime \prime}(z)= \begin{cases}H^{\prime \prime}(z), & \text { if } z \leq \bar{x}_{1} \text { and } z \neq R_{j}, \\ H^{\prime \prime}(z)+\alpha \int_{0}^{z-\bar{x}_{1}} H^{\prime \prime}(z-b) \phi(b) d b, & \text { if } z>\bar{x}_{1} \text { and } z \neq R_{j},\end{cases}
$$

where $j=1, \ldots, m$. We obtain

$$
F_{1+}^{\prime \prime}\left(R_{i}\right)= \begin{cases}H_{+}^{\prime \prime}\left(R_{i}\right), & \text { if } R_{i} \leq \bar{x}_{1}, \\ H_{+}^{\prime \prime}\left(R_{i}\right)+\alpha \int_{0}^{R_{i}-\bar{x}_{1}} H^{\prime \prime}\left(R_{i}-b\right) \phi(b) d b, & \text { if } R_{i}>\bar{x}_{1}\end{cases}
$$

and

$$
F_{1-}^{\prime \prime}\left(R_{i}\right)= \begin{cases}H_{-}^{\prime \prime}\left(R_{i}\right), & \text { if } R_{i} \leq \bar{x}_{1}, \\ H_{-}^{\prime \prime}\left(R_{i}\right)+\alpha \int_{0}^{R_{i}-\bar{x}_{1}} H^{\prime \prime}\left(R_{i}-b\right) \phi(b) d b, & \text { if } R_{i}>\bar{x}_{1}\end{cases}
$$

We have $F_{1}^{\prime \prime}(z) \geq 0$ on $\left(R_{i-1}, R_{i}\right)$ for all $i$.

Proof. As the method we used in Lemma 2.3 we can calculate a second derivative of $F_{1}(z)$. It also follows from the inequality $H^{\prime \prime}(z) \geq 0$ on $\left(R_{i-1}, R_{i}\right)$ that $F_{1}(z)$ is a convex function on $\left[R_{i-1}, R_{i}\right]$ and hence our lemma is proved.

LEMMA 2.5. We have

$$
\lim _{z \rightarrow-\infty} F_{1}^{\prime}(z)<0, \quad \lim _{z \rightarrow \infty} F_{1}^{\prime}(z)>c .
$$

Proof. By (2.2) and Lemma 2.3 we see that

$$
\lim _{z \rightarrow-\infty} F_{1}^{\prime}(z)=\lim _{z \rightarrow-\infty}\left(H^{\prime}(z)-\alpha c\right)<0 .
$$


If $0 \leq b \leq z-\bar{x}_{1}$, then $z-b \geq \bar{x}_{1}$ and therefore $H^{\prime}(z-b) \geq 0$. It thus follows that

$$
\begin{aligned}
\lim _{z \rightarrow \infty} F_{1}^{\prime}(z) & =\lim _{z \rightarrow \infty}\left(H^{\prime}(z)-\alpha c+\alpha \int_{0}^{z-\bar{x}_{1}} H^{\prime}(z-b) \phi(b) d b\right) \\
& =\lim _{z \rightarrow \infty} H^{\prime}(z)-\alpha c+\alpha \int_{0}^{\infty} \lim _{z \rightarrow \infty} H^{\prime}(z) \phi(b) d b \\
& =\lim _{z \rightarrow \infty} H^{\prime}(z)(1+\alpha)-\alpha c>c .
\end{aligned}
$$

This completes the proof.

Now we have the following fundamental Theorem .

Theorem 2.6. Assume that $1 \leq k \leq N-1$. Then there is a real number $\bar{x}_{k}$ such that $\bar{x}_{k}=\inf \left\{z \mid F_{(k-1)+}^{\prime}(z) \geq 0\right\}$. We have the following statemens:

1.

$$
f_{k}(x)= \begin{cases}-c x+F_{k-1}\left(\bar{x}_{k}\right), & \text { if } x \leq \bar{x}_{k} \\ -c x+F_{k-1}(x), & \text { if } x \geq \bar{x}_{k}\end{cases}
$$

In particular $f_{k}(x)$ is continuous on $\boldsymbol{R}$.

2.

$$
F_{k}(z)= \begin{cases}H(z)-\alpha c z+\alpha\left\{F_{k-1}\left(\bar{x}_{1}\right)+c E(B)\right\}, & \text { if } z \leq \bar{x}_{k}, \\ H(z)-\alpha c z+\alpha \int_{0}^{z-\bar{x}_{k}}\left\{F_{k-1}(z-b)-F_{k-1}\left(\bar{x}_{k}\right)\right\} \phi(b) d b & \\ +\alpha\left\{F_{k-1}\left(\bar{x}_{k}\right)+c E(B)\right\}, & \text { if } z>\bar{x}_{k} .\end{cases}
$$

Furthermore $F_{k}(z)$ is continuous on $\boldsymbol{R}$.

3. $F_{k}(z)$ has a derivative on $\left[R_{i-1}, R_{i}\right]$ for all $i(1 \leq i \leq m+1)$, given by

$$
F_{k}^{\prime}(z)= \begin{cases}H^{\prime}(z)-\alpha c, & \text { if } z \leq \bar{x}_{k} \text { and } z \neq R_{j}, \\ H^{\prime}(z)-\alpha c+\alpha \int_{0}^{z-\bar{x}_{k}} F_{k-1}^{\prime}(z-b) \phi(b) d b, & \text { if } z>\bar{x}_{k} \text { and } z \neq R_{j},\end{cases}
$$

where $j=1, \ldots, m$. We have

$$
F_{k+}^{\prime}\left(R_{i}\right)= \begin{cases}H_{+}^{\prime}\left(R_{i}\right)-\alpha c, & \text { if } R_{i} \leq \bar{x}_{k} \\ H_{+}^{\prime}\left(R_{i}\right)-\alpha c+\alpha \int_{0}^{R_{i}-\vec{x}_{k}} F_{k-1}^{\prime}\left(R_{i}-b\right) \phi(b) d b, & \text { if } R_{i}>\bar{x}_{k}\end{cases}
$$

and

$$
F_{k-}^{\prime}\left(R_{i}\right)= \begin{cases}H_{-}^{\prime}\left(R_{i}\right)-\alpha c, & \text { if } R_{i} \leq \bar{x}_{k} \\ H_{-}^{\prime}\left(R_{i}\right)-\alpha c+\alpha \int_{0}^{R_{i}-\bar{x}_{k}} F_{k-1}^{\prime}\left(R_{i}-b\right) \phi(b) d b, & \text { if } R_{i}>\bar{x}_{k}\end{cases}
$$

in particular we see $F_{k-}^{\prime}\left(R_{i}\right) \leq F_{k+}^{\prime}\left(R_{i}\right)$ for all $i \quad(1 \leq i \leq m)$ 
4. $F_{k}(z)$ has a second continuous derivative on $\left[R_{i-1}, R_{i}\right]$ for all $i(1 \leq i \leq m+1)$, given

$$
F_{k}^{\prime \prime}(z)= \begin{cases}H^{\prime \prime}(z), & \text { if } z \leq \bar{x}_{k} \text { and } z \neq R_{j}, \\ H^{\prime \prime}(z)+\alpha \int_{0}^{z-\bar{x}_{k}} F_{k-1}^{\prime \prime}(z-b) \phi(b) d b, & \text { if } z>\bar{x}_{k} \text { and } z \neq R_{j}\end{cases}
$$

where $j=1, \ldots, m$. We obtain

$$
F_{k+}^{\prime \prime}\left(R_{i}\right)= \begin{cases}H_{+}^{\prime \prime}\left(R_{i}\right), & \text { if } R_{i} \leq \bar{x}_{k} \\ H_{+}^{\prime \prime}\left(R_{i}\right)+\alpha \int_{0}^{R_{i}-\bar{x}_{k}} F_{k-1}^{\prime \prime}\left(R_{i}-b\right) \phi(b) d b, & \text { if } R_{i}>\bar{x}_{k}\end{cases}
$$

and

$$
F_{k-}^{\prime \prime}\left(R_{i}\right)= \begin{cases}H_{-}^{\prime \prime}\left(R_{i}\right), & \text { if } R_{i} \leq \bar{x}_{k}, \\ H_{-}^{\prime \prime}\left(R_{i}\right)+\alpha \int_{0}^{R_{i}-\bar{x}_{k}} F_{k-1}^{\prime \prime}\left(R_{i}-b\right) \phi(b) d b, & \text { if } R_{i}>\bar{x}_{k} .\end{cases}
$$

We have $F_{k}^{\prime \prime}(z) \geq 0$ on $\left(R_{i-1}, R_{i}\right)$ for all $i$.

5. $F_{k+}^{\prime}(z)$ is a non-decreasing function on the set $\boldsymbol{R}$ of all real numbers.

6. We have

$$
\lim _{z \rightarrow-\infty} F_{k}^{\prime}(z)<0, \quad \lim _{z \rightarrow \infty} F_{k}^{\prime}(z)>c
$$

Proof. If $k=1$, our theorem follows from Lemma 2.1 through Lemma 2.5. Since we set $F_{0}(z)=H(z)$, the properties of the function $F_{1}(z)$ is gotten from those of $F_{0}(z)$. That is, we know by (2.6) and (2.7) that

$$
\begin{aligned}
& f_{2}(x)=\min _{z \geq x}\left\{-c x+F_{1}(z)\right\} \\
& F_{2}(z)=H(z)+\alpha \int_{0}^{\infty} f_{2}(z-b) \phi(b) d b .
\end{aligned}
$$

We also see by Lemma 2.1 through Lemma 2.5 that the same assumptions which we suppose on the function $H(z)$ hold on $F_{1}(z)$. That is, $F_{1}(z)$ is continuous on $(-\infty, \infty)$ and has a second derivative on the interval $\left[R_{i-1}, R_{i}\right]$ for all $i(1 \leq i \leq m)$. And also $F_{1}(z)$ is a convex function on $\left[R_{i-1}, R_{i}\right]$ for all $i$ and we have

$$
F_{1-}^{\prime}\left(R_{i}\right) \leq F_{1+}^{\prime}\left(R_{i}\right) \text { for } i(1 \leq i \leq m)
$$

and

$$
\lim _{z \rightarrow-\infty} F_{1}^{\prime}(z)<0, \quad \lim _{z \rightarrow \infty} F_{1}^{\prime}(z)>c .
$$

Thus we can repeat the proof of lemmata to conclude that our theorem holds in the case $k=2$. We are able to proceed to prove it in the case $k \geq 3$ and we complete our proof.

The following inequalities is valid to decide the sign of $F_{k}^{\prime}\left(R_{i}\right)(1 \leq k \leq N-1,1 \leq$ $i \leq m$ ) (cf. Theorem 1 in Kodama and Sakaguchi, 2001b). 
ThEOREM 2.7. We have

$$
\begin{aligned}
& H^{\prime}(z)-\alpha c \leq F_{k}^{\prime}(z) \leq H^{\prime}(z)-\alpha c+\alpha F_{k-1}^{\prime}(z), \quad \text { if } z \geq \bar{x}_{k} \text { and } z \neq R_{i}, \\
& H_{+}^{\prime}\left(R_{i}\right)-\alpha c \leq F_{k+}^{\prime}\left(R_{i}\right) \leq H_{+}^{\prime}\left(R_{i}\right)-\alpha c+\alpha F_{(k-1)+}^{\prime}\left(R_{i}\right), \quad \text { if } R_{i} \geq \bar{x}_{k}, \\
& H_{-}^{\prime}\left(R_{i}\right)-\alpha c \leq F_{k-}^{\prime}\left(R_{i}\right) \leq H_{-}^{\prime}\left(R_{i}\right)-\alpha c+\alpha F_{(k-1)-}^{\prime}\left(R_{i}\right), \quad \text { if } R_{i} \geq \bar{x}_{k},
\end{aligned}
$$

for $1 \leq k \leq N-1$.

Proof. Suppose that $\bar{x}_{k} \leq z$ and $z \neq R_{i}$. It follows from 3 in Theorem 2.6 that

$$
F_{k}^{\prime}(z)=H^{\prime}(z)-\alpha c+\alpha \int_{0}^{z-\bar{x}_{k}} F_{k-1}^{\prime}(z-b) \phi(b) d b .
$$

Note that $0 \leq b \leq z-\bar{x}_{k}$ if and only if $\bar{x}_{k} \leq z-b \leq z$. Since $F_{k-1}^{\prime}(z)$ is a non-decreasing, we know that

$$
0 \leq F_{k-1}^{\prime}\left(\bar{x}_{k}\right) \leq F_{k-1}^{\prime}(z-b) \leq F_{k-1}^{\prime}(z) \quad \text { for } 0 \leq b \leq z-\bar{x}_{k}
$$

which implies

$$
0 \leq \int_{0}^{z-\bar{x}_{k}} F_{k-1}^{\prime}(z-b) \phi(b) d b \leq \int_{0}^{z-\bar{x}_{k}} F_{k-1}^{\prime}(z) \phi(b) d b \leq F_{k-1}^{\prime}(z) .
$$

Consequently we obtain

$$
H^{\prime}(z)-\alpha c \leq F_{k}^{\prime}(z) \leq H^{\prime}(z)-\alpha c+\alpha F_{k-1}^{\prime}(z)
$$

and, by 3 in Theorem 2.6, we also have

$$
\begin{aligned}
& H_{+}^{\prime}\left(R_{i}\right)-\alpha c \leq F_{k+}^{\prime}\left(R_{i}\right) \leq H_{+}^{\prime}\left(R_{i}\right)-\alpha c+\alpha F_{(k-1)+}^{\prime}\left(R_{i}\right), \\
& H_{-}^{\prime}\left(R_{i}\right)-\alpha c \leq F_{k-}^{\prime}\left(R_{i}\right) \leq H_{-}^{\prime}\left(R_{i}\right)-\alpha c+\alpha F_{(k-1)-}^{\prime}\left(R_{i}\right) .
\end{aligned}
$$

We complete the proof.

\section{Optimal policies}

We wish to find the sequence $\bar{x}_{1}, \ldots, \bar{x}_{N}$ to get optimal policies in the system of the probabilistic dynamic inventory problem. We studied in Kodama and Sakaguchi (2001a), Kodama and Sakaguchi (2001b) and Kodama and Sakaguchi (2001c) the interval $\left[R_{p-1}\right.$, $R_{p}$ ] which contains $\bar{x}_{k}$ under the assumption that the function $H^{\prime}(z)$ is continuous. The interval may be found if we could seek the conditons $F_{k-1}^{\prime}\left(R_{p-1}\right)<0$ and $F_{k-1}^{\prime}\left(R_{p}\right) \geq 0$. Although functions $F_{i}^{\prime}(z)$ may be discontinuous in this paper, we have similar results considering the right derivatives $F_{i+}^{\prime}(z)(1 \leq i \leq N-1)$ and we see them in the following theorem (cf. Theorem 2 in Kodama and Sakaguchi, 2001c).

THEOREM 3.1. Let $R$ be a real number. Then the following statements hold:

1. If $H_{+}^{\prime}(R) \geq \alpha c$, then $F_{k+}^{\prime}(R) \geq 0$ for all $k 1 \leq k \leq N-1$.

2. If $1 \leq k \leq N-1$ and $F_{(k-1)+}^{\prime}(R) \leq 0$, then $F_{k+}^{\prime}(R)=H_{+}^{\prime}(R)-\alpha c$. 
3. If $1 \leq k \leq N-1, H_{+}^{\prime}(R)<\alpha c$ and $F_{(k-1)+}^{\prime}(R) \leq 0$, then $F_{k+}^{\prime}(R)<0$.

4. If $H_{+}^{\prime}(R)<0$, then $F_{k+}^{\prime}(R)<0$ for all $k 1 \leq k \leq N-1$.

5. If $1 \leq k \leq N-1$ and $H_{+}^{\prime}(R)<\frac{\alpha c\left(1+\alpha+\cdots+\alpha^{k-1}\right)}{1+\alpha+\cdots+\alpha^{k}}$, then $F_{k+}^{\prime}(R)<0$.

6. If $1 \leq k \leq N-1$ and $H_{+}^{\prime}(R)=\frac{\alpha c\left(1+\alpha+\cdots+\alpha^{k-1}\right)}{1+\alpha+\cdots+\alpha^{k}}$, then $F_{k+}^{\prime}(R) \leq 0$.

Proof. 1. If $R \leq \bar{x}_{k}$, then this is a direct consequence of 3 in Theorem 2.6. If $R>\bar{x}_{k}$, then it is also clear by Theorem 2.7 .

2. In fact, if $F_{(k-1)+}^{\prime}(R)<0$, then $R<\bar{x}_{k}$, and hence $F_{k+}^{\prime}(R)=H_{+}^{\prime}(R)-\alpha c$ by 3 in Theorem 2.6. If $F_{(k-1)+}^{\prime}(R)=0$, then $\bar{x}_{k} \leq R$. It follows from Theorem 2.7 that

$$
H_{+}^{\prime}(R)-\alpha c \leq F_{k+}^{\prime}(R) \leq H_{+}^{\prime}(R)-\alpha c+\alpha F_{(k-1)+}^{\prime}(R) .
$$

Substituting $F_{(k-1)+}^{\prime}(R)=0$ we have $F_{k+}^{\prime}(R)=H_{+}^{\prime}(R)-\alpha c$.

3. This easily follows from 2 .

4. The statement 3 implies 4 immediately.

5. We see that $H_{+}^{\prime}(R)<\alpha c$. Therefore by 3 we may assume that $F_{i+}^{\prime}(R)>0$ for all $i \cdot 0 \leq i \leq k-1$. Hence it follows that $\bar{x}_{i+1} \leq R$. By Theorem 2.7 we have

$$
F_{(i+1)+}^{\prime}(R) \leq H_{+}^{\prime}(R)-\alpha c+\alpha F_{i+}^{\prime}(R) \quad \text { for all } i 0 \leq i \leq k-1 .
$$

By these inequalities and our assumption we obtain

$$
F_{k+}^{\prime}(R) \leq H_{+}^{\prime}(R)\left(1+\alpha+\cdots+\alpha^{k}\right)-\alpha c\left(1+\alpha+\cdots+\alpha^{k-1}\right)<0 .
$$

6. We are also able to prove this by the same method of 5 that $F_{k+}^{\prime}(R) \leq 0$, because

$$
H_{+}^{\prime}(R)\left(1+\alpha+\cdots+\alpha^{k}\right)-\alpha c\left(1+\alpha+\cdots+\alpha^{k-1}\right)=0 .
$$

We complete our proof.

CoROLlaRY 3.2. Let $p$ be an integer with $1 \leq p \leq m$. Then we have:

1. If $H_{+}^{\prime}\left(R_{p}\right) \geq \alpha c$, then $\bar{x}_{k} \leq R_{p}$ for all $k 1 \leq k \leq N$.

2. If $H_{+}^{\prime}\left(R_{p}\right)<0$, then $R_{p}<\bar{x}_{k}$ for all $k 1 \leq k \leq N$.

3. If $H_{+}^{\prime}\left(R_{p}\right)<\frac{\alpha c\left(1+\alpha+\cdots+\alpha^{l-1}\right)}{1+\alpha+\cdots+\alpha^{l}}$, then $R_{p}<\bar{x}_{k}$ for all $k l+1 \leq k \leq N$. 
Proof. These assertions are followed from Theorem 3.1, because $\bar{x}_{k} \leq R_{p}$ if and only if $F_{(k-1)+}^{\prime}\left(R_{p}\right) \geq 0$, and the proof is complete.

For the purpose of calculating $\bar{x}_{k}$ we wish to get conditions that support us how to choose some functions $H_{i}(z)$ among the functions $H_{1}(z), \ldots, H_{m+1}(z)$. Since there exist many complicated cases, we only consider simpler cases.

At first we know that

$$
H_{+}^{\prime}\left(R_{1}\right) \leq H_{+}^{\prime}\left(R_{2}\right) \leq \cdots \leq H_{+}^{\prime}\left(R_{m}\right) .
$$

The corollary shows the following four particular cases:

Case A. If $H_{+}^{\prime}\left(R_{1}\right) \geq \alpha c$, then $\bar{x}_{k} \leq R_{1}$ for all $k \quad 1 \leq k \leq N$.

Case B. If $H_{+}^{\prime}\left(R_{m}\right)<0$, then $R_{m}<\bar{x}_{k}$ for all $k \quad 1 \leq k \leq N$.

Case C. If $H_{+}^{\prime}\left(R_{p-1}\right)<0$ and $H_{+}^{\prime}\left(R_{p}\right) \geq \alpha c$, then $R_{p-1}<\bar{x}_{k} \leq R_{p}$ for all $k 1 \leq k \leq N$.

Case $\mathrm{D}_{l}$. If $H_{+}^{\prime}\left(R_{p-1}\right)<0, F_{(l-1)+}^{\prime}\left(R_{p}\right) \geq 0$ and $H_{+}^{\prime}\left(R_{m}\right)<\frac{\alpha c\left(1+\alpha+\cdots+\alpha^{l-1}\right)}{1+\alpha+\cdots+\alpha^{l}}$, then $R_{p-1}<\bar{x}_{k} \leq R_{p}$ for all $k \quad 1 \leq k \leq l$ and $R_{m}<\bar{x}_{k}$ for all $k l+1 \leq k \leq N$.

When $m=1$, the optimal policies are studied at the case $\mathrm{D}_{l}$ in Kodama (1998a).

Now we shall consider the probabilistic multi-period inventory model with zero delivery lag, backlogging of demand and linear purchasing cost $[c(y)=c y]$. Let $-c x+$ $H(z)$ denote the expected one-period loss, given $z$ is an amount on hand after an order is placed and let $f_{k}(x)$ denote the minimum total discount expected loss for period $1,2, \ldots, k$ where $x$ is the initial stock level. From the principal of optimality, each $f_{k}(x)$ satisfies the functional equation (2.5) and (2.6). Then the optimal policy in our system of the probabilistic dynamic inventory problem is the following:

$$
\text { If } x<\bar{x}_{k} \text {, then order }\left(\bar{x}_{k}-x\right) \text {, otherwise do not order. }
$$

Now we study how to get the sequence $\bar{x}_{1}, \bar{x}_{2}, \cdots, \bar{x}_{N}$ in simple cases. In general we have the following proposition (cf. Theorem 2.2 in Kodama, 1998a).

Proposition 3.3. We have

$$
\bar{x}_{1} \leq \bar{x}_{2} \leq \cdots \leq \bar{x}_{N}
$$

Proof. We prove $\bar{x}_{p} \leq \bar{x}_{p+1}$ by induction on $p$. By the definition of $\bar{x}_{1}, H_{+}^{\prime}(z)$ is negative for $z<\bar{x}_{1}$ and therefore if $z<\bar{x}_{1}$, then $F_{1+}^{\prime}(z)=H_{+}^{\prime}(z)-\alpha c<0$. This impies $\bar{x}_{1} \leq \bar{x}_{2}$. Now we may assume $p \geq 2$. It is shown that $F_{(p-1)+}^{\prime}(z)<0$ for $z<\bar{x}_{p}$. Therefore if $z<\bar{x}_{p-1}$, then it follows from 3 in Theorem 2.6 that $F_{(p-1)+}^{\prime}(z)=$ $H_{+}^{\prime}(z)-\alpha c<0$.

By the inductive hypothesis we obtain $\bar{x}_{p-1} \leq \bar{x}_{p}$. Suppose that $\bar{x}_{p-1} \leq z<\bar{x}_{p}$. Then we have by 3 in Theorem 2.6

$$
F_{(p-1)+}^{\prime}(z)=H_{+}^{\prime}(z)-\alpha c+\alpha \int_{0}^{z-\bar{x}_{p-1}} F_{p-2}^{\prime}(z-b) \phi(b) d b<0 .
$$

If $0 \leq b \leq z-\bar{x}_{p-1}$ and $z-b \neq R_{j}$, then $\bar{x}_{p-1} \leq z-b \leq z<\bar{x}_{p}$, and so $F_{p-2}^{\prime}(z-b) \geq 0$ because $F_{p-2}^{\prime}(z)$ is not decreasing and $F_{p-2}^{\prime}\left(\bar{x}_{p-1}\right) \geq 0$. This fact implies

$$
\int_{0}^{z-\tilde{x}_{p-1}} F_{p-2}^{\prime}(z-b) \phi(b) d b \geq 0
$$


and hence we obtain by (3.1) that $H_{+}^{\prime}(z)-\alpha c<0$ for $\bar{x}_{p-1} \leq z<\bar{x}_{p}$.

We have showed that $F_{p+}^{\prime}(z)=H_{+}^{\prime}(z)-\alpha c<0$ for $z<\bar{x}_{p}$. Accordingly we conclude that $\bar{x}_{p} \leq \bar{x}_{p+1}$, and this proposition is proved.

The following proposition is obtained from 3 in Theorem 2.6.

Proposition 3.4. (Section 5 in Kodama, 1998a) We have for $z \geq \bar{x}_{k}$ with $z \neq$ $R_{i} \quad(1 \leq i \leq m)$

$$
\begin{aligned}
F_{k}^{\prime}(z)= & H^{\prime}(z)-\alpha c+\alpha \int_{0}^{z-\bar{x}_{k}}\left(H^{\prime}\left(z-b_{k}\right)-\alpha c\right) \phi\left(b_{k}\right) d b_{k} \\
& +\alpha^{2} \int_{0}^{z-\bar{x}_{k}} \int_{0}^{z-b_{k}-\bar{x}_{k-1}}\left(H^{\prime}\left(z-b_{k}-b_{k-1}\right)-\alpha c\right) \phi\left(b_{k-1}\right) \phi\left(b_{k}\right) d b_{k-1} d b_{k} \\
& +\alpha^{3} \int_{0}^{z-\bar{x}_{k}} \int_{0}^{z-b_{k}-\bar{x}_{k-1}} \int_{0}^{z-b_{k}-b_{k-1}-\bar{x}_{k-2}} \\
& \left.\quad+H^{\prime}\left(z-b_{k}-b_{k-1}-b_{k-2}\right)-\alpha c\right) \phi\left(b_{k-2}\right) \phi\left(b_{k-1}\right) \phi\left(b_{k}\right) d b_{k-2} d b_{k-1} d b_{k} \\
& +\alpha^{k-1} \int_{0}^{z-\bar{x}_{k}} \int_{0}^{z-b_{k}-\bar{x}_{k-1}} \cdots \int_{0}^{z-b_{k}-b_{k-1}-\cdots-b_{3}-\bar{x}_{2}} \\
& \left(H^{\prime}\left(z-b_{k}-b_{k-1}-\cdots-b_{2}\right)-\alpha c\right) \phi\left(b_{2}\right) \phi\left(b_{3}\right) \cdots \phi\left(b_{k}\right) d b_{2} d b_{3} \cdots d b_{k} \\
& +\alpha^{k} \int_{0}^{z-\bar{x}_{k}} \int_{0}^{z-b_{k}-\bar{x}_{k-1}} \cdots \int_{0}^{z-b_{k}-b_{k-1}-\cdots-b_{2}-\bar{x}_{1}} \\
& H^{\prime}\left(z-b_{k}-b_{k-1}-\cdots-b_{1}\right) \phi\left(b_{1}\right) \phi\left(b_{2}\right) \cdots \phi\left(b_{k}\right) d b_{1} d b_{2} \cdots d b_{k} .
\end{aligned}
$$

Case A. Assume $H_{+}^{\prime}\left(R_{1}\right)=H_{2+}^{\prime}\left(R_{1}\right) \geq \alpha c$. Then it follows that $\bar{x}_{k} \leq R_{1}$ for all $k(1 \leq k \leq N)$.

Proposition 3.5. Assume $H_{1-}^{\prime}\left(R_{1}\right)<0$ and $H_{2+}^{\prime}\left(R_{1}\right) \geq \alpha$. Then we have $\bar{x}_{k}=$ $R_{1}$ for all $k(1 \leq k \leq N)$.

Proof. Since $H_{1-}^{\prime}\left(R_{1}\right)<0, H^{\prime}(z)$ is negative for $z<R_{1}$. Whence $\bar{x}_{1} \geq R_{1}$, and we complete our proof by Proposition 3.3 and the condition in Case A.

Proposition 3.6. Suppose $H_{2+}^{\prime}\left(R_{1}\right) \geq \alpha$ c. If $\bar{x}_{p}=R_{1}$ for some $p(1 \leq p \leq N-1)$, then $\bar{x}_{k}=R_{1}$ for all $k$ with $p+1 \leq k \leq N$.

Proof. This is also clear by Proposition 3.3 and the condition in Case A.

Let $\bar{x}_{k+1}<R_{1}$. Then $\bar{x}_{k+1}$ is a solution of the equation $F_{k}^{\prime}(z)=0$, precisely it is the minimal solution of its equation. Because of $F_{0}^{\prime}(z)=H_{1}^{\prime}(z)$ for $z<R_{1}$, we may find $\bar{x}_{1}$ to solve the equation $H_{1}^{\prime}(z)=0$.

For $\bar{x}_{1} \leq z<R_{1}$,

$$
F_{1}^{\prime}(z)=H_{1}^{\prime}(z)-\alpha c+\alpha \int_{0}^{z-\bar{x}_{1}} H_{1}^{\prime}\left(z-b_{1}\right) \phi\left(b_{1}\right) d b_{1} .
$$


Thus we can get $\bar{x}_{2}$ to solve the equation $F_{1}^{\prime}(z)=0$. It follows from Proposition 3.4 that the functions $F_{k}^{\prime}(z)$ for $\bar{x}_{k} \leq z<R_{1}$ can be written by replacing $H^{\prime}(z)$ with $H_{1}^{\prime}(z)$, and we keep on this step.

Case B. Suppose $H_{+}^{\prime}\left(R_{m}\right)<0$. Then we have $R_{m}<\bar{x}_{k}$ for all $k 1 \leq k \leq N$.

In this case there is a number $\bar{x}_{1}$ that is a solution of the equation $\bar{H}_{m+1}^{\prime}(z)=0$ by the fact that $F_{0}^{\prime}(z)=H_{m+1}^{\prime}(z)$ for $z>R_{m}$. Replacing all the function $H^{\prime}(z)$ in the equation of Proposition 3.4 with $H_{m+1}^{\prime}(z)$, we are able to write $F_{k}^{\prime}(z)$ for $z>R_{m}$ by using only the function $H_{m+1}^{\prime}(z)$.

Case C. Assume that $H_{+}^{\prime}\left(R_{p-1}\right)<0$ and $H_{+}^{\prime}\left(R_{p}\right) \geq \alpha c$. Then it follows that $R_{p-1}<\bar{x}_{k} \leq R_{p}$ for all $k 1 \leq k \leq N$. Applying the properties in cases $\mathrm{A}$ and $\mathrm{B}$ to this case we know the following statements:

1. If $H_{-}^{\prime}\left(R_{p}\right)<0$, then $\bar{x}_{k}=R_{p}$ for all $k(1 \leq k \leq N)$.

2. If $\bar{x}_{k}=R_{p}$ for some $p(1 \leq p \leq N-1)$, then $\bar{x}_{k}=R_{p}$ for all $k(p+1 \leq k \leq N)$.

3. If $\bar{x}_{k+1}<R_{p}$, then $R_{p-1}<\bar{x}_{1}<R_{p}$. Hence we may find $\bar{x}_{1}$ to solve the equation $H_{p}^{\prime}(z)=0$. Replacing all the function $H^{\prime}(z)$ in Proposition 3.4 with $H_{p}^{\prime}(z)$, the functions $F_{k}^{\prime}(z)\left(R_{p-1}<z<R_{p}\right)$ can be expressed in terms of the function $H_{p}^{\prime}(z)$. For the sake of finding $\bar{x}_{k+1}$, it is enough to solve the equations $F_{i}^{\prime}(z)=0 \quad(1 \leq$ $i \leq k)$ successively.

Case $\mathbf{D}_{l}$. Suppose that $H_{+}^{\prime}\left(R_{p-1}\right)<0, F_{(l-1)+}^{\prime}\left(R_{p}\right) \geq 0$ and $H_{+}^{\prime}\left(R_{m}\right)<$ $\frac{\alpha c\left(1+\alpha+\cdots+\alpha^{l-1}\right)}{1+\alpha+\cdots+\alpha^{l}}$. Then we have $R_{p-1}<\bar{x}_{k} \leq R_{p}$ for all $k 1 \leq k \leq l$ and $R_{m}<\bar{x}_{k}$ for all $k l+1 \leq k \leq N$.

In this case it is necessary to change the $H_{i}^{\prime}(z)$ in order to express $F_{k}^{\prime}(z)$. We write them explicitly. At first solve the equation $H_{p}^{\prime}(z)=0$ to get $\bar{x}_{1}$. Next for $2 \leq k \leq l-1$ and $\bar{x}_{k} \leq z<R_{p}$, we have by Proposition 3.4

$$
\begin{aligned}
F_{k}^{\prime}(z)= & H_{p}^{\prime}(z)-\alpha c+\alpha \int_{0}^{z-\bar{x}_{k}}\left(H_{p}^{\prime}\left(z-b_{k}\right)-\alpha c\right) \phi\left(b_{k}\right) d b_{k} \\
& +\alpha^{2} \int_{0}^{z-\bar{x}_{k}} \int_{0}^{z-b_{k}-\bar{x}_{k-1}}\left(H_{p}^{\prime}\left(z-b_{k}-b_{k-1}\right)-\alpha c\right) \phi\left(b_{k-1}\right) \phi\left(b_{k}\right) d b_{k-1} d b_{k} \\
& +\alpha^{3} \int_{0}^{z-\bar{x}_{k}} \int_{0}^{z-b_{k}-\bar{x}_{k-1}} \int_{0}^{z-b_{k}-b_{k-1}-\bar{x}_{k-2}} \\
& +\cdots \cdots\left(H_{p}^{\prime}\left(z-b_{k}-b_{k-1}-b_{k-2}\right)-\alpha c\right) \phi\left(b_{k-2}\right) \phi\left(b_{k-1}\right) \phi\left(b_{k}\right) d b_{k-2} d b_{k-1} d b_{k} \\
& +\alpha^{k-1} \int_{0}^{z-\bar{x}_{k}} \int_{0}^{z-b_{k}-\bar{x}_{k-1}} \cdots \int_{0}^{z-b_{k}-b_{k-1}-\cdots-b_{3}-\bar{x}_{2}} \\
& \left(H_{p}^{\prime}\left(z-b_{k}-b_{k-1}-\cdots-b_{2}\right)-\alpha c\right) \phi\left(b_{2}\right) \phi\left(b_{3}\right) \cdots \phi\left(b_{k}\right) d b_{2} d b_{3} \cdots d b_{k} \\
& +\alpha^{k} \int_{0}^{z-\bar{x}_{k}} \int_{0}^{z-b_{k}-\bar{x}_{k-1}} \cdots \int_{0}^{z-b_{k}-b_{k-1}-\cdots-b_{2}-\bar{x}_{1}} \\
& H_{p}^{\prime}\left(z-b_{k}-b_{k-1}-\cdots-b_{1}\right) \phi\left(b_{1}\right) \phi\left(b_{2}\right) \cdots \phi\left(b_{k}\right) d b_{1} d b_{2} \cdots d b_{k} .
\end{aligned}
$$


We may continue to find a solution $\bar{x}_{k+1}$ of the equation $F_{k}^{\prime}(z)=0, \bar{x}_{k} \leq z<R_{p}$ for $2 \leq k \leq l-1$. If we could not find any solution, then $\bar{x}_{k+1}=R_{p}$.

Next we shall show $F_{l}^{\prime}(z)$ by $H_{i}^{\prime}(z)$, however it is complicated. For the simplicity we introduce the functions $h_{l}(z), h_{l-1}(z), h_{l-2}(z), \ldots, h_{2}(z), h_{1}(z)$ for $z>R_{m}$ as follows.

$$
\begin{aligned}
h_{l-s}(z)= & \int_{0}^{z-\bar{x}_{l}} \int_{0}^{z-b_{l}-\bar{x}_{l-1}} \cdots \int_{0}^{z-b_{l}-b_{l-1} \cdots-b_{l-s+1}-\bar{x}_{l-s}} \\
& \left(H^{\prime}\left(z-b_{l}-b_{l-1} \cdots-b_{l-s}\right)-\alpha c\right) \phi\left(b_{l-s}\right) \cdots \phi\left(b_{l-1}\right) \phi\left(b_{l}\right) d b_{l-s} \cdots d b_{l-1} d b_{l} \\
h_{1}(z)= & \int_{0}^{z-\bar{x}_{l}} \int_{0}^{z-b_{l}-\bar{x}_{l-1}} \cdots \int_{0}^{z-b_{l}-b_{l-1}-\cdots-b_{2}-\bar{x}_{1}} \\
& H^{\prime}\left(z-b_{l}-b_{l-1}-\cdots-b_{1}\right) \phi\left(b_{1}\right) \phi\left(b_{2}\right) \cdots \phi\left(b_{l}\right) d b_{1} d b_{2} \cdots d b_{l} .
\end{aligned}
$$

Then we obtain

$$
\begin{aligned}
h_{l}(z)= & \int_{0}^{z-\bar{x}_{l}} H^{\prime}\left(z-b_{l}\right) \phi\left(b_{l}\right) d b_{l}-\alpha c \int_{0}^{z-\bar{x}_{l}} \phi\left(b_{l}\right) d b_{l} \\
= & \int_{0}^{z-R_{m}} H^{\prime}\left(z-b_{l}\right) \phi\left(b_{l}\right) d b_{l}+\sum_{t=p}^{m-1} \int_{z-R_{m+p-t}}^{z-R_{m+p-t-1}} H^{\prime}\left(z-b_{l}\right) \phi\left(b_{l}\right) d b_{l} \\
& +\int_{z-R_{p}}^{z-\bar{x}_{l}} H^{\prime}\left(z-b_{l}\right) \phi\left(b_{l}\right) d b_{l}-\alpha c \int_{0}^{z-\bar{x}_{l}} \phi\left(b_{l}\right) d b_{l} \\
= & \int_{0}^{z-R_{m}} H_{m+1}^{\prime}\left(z-b_{l}\right) \phi\left(b_{l}\right) d b_{l}+\sum_{t=p}^{m-1} \int_{z-R_{m+p-t}}^{z-R_{m+p-t-1}} H_{m+p-t}^{\prime}\left(z-b_{l}\right) \phi\left(b_{l}\right) d b_{l} \\
& +\int_{z-R_{p}}^{z-\bar{x}_{l}} H_{p}^{\prime}\left(z-b_{l}\right) \phi\left(b_{l}\right) d b_{l}-\alpha c \int_{0}^{z-\bar{x}_{l}} \phi\left(b_{l}\right) d b_{l} .
\end{aligned}
$$

Let $1 \leq s \leq l-1$. We set

$$
\begin{aligned}
K_{l-s}= & \left\{\left(b_{l-s}, b_{l-s+1}, \ldots, b_{l}\right) \mid 0 \leq b_{l-n} \leq z-\sum_{j=0}^{n-1} b_{l-j}-\bar{x}_{l-n}, 0 \leq n \leq s\right\}, \\
K_{l-s p}= & \left\{\left(b_{l-s}, b_{l-s+1}, \ldots, b_{l}\right) \mid z-R_{p} \leq \sum_{j=0}^{s} b_{l-s+j} \leq z-\bar{x}_{l-s},\right. \\
& \left.\sum_{j=0}^{n} b_{l-n+j} \leq z-\bar{x}_{l-n}(0 \leq n \leq s-1), b_{l-s+n} \geq 0(0 \leq n \leq s)\right\}, \\
K_{l-s i}= & \left\{\left(b_{l-s}, b_{l-s+1}, \ldots, b_{l}\right) \mid z-R_{i} \leq \sum_{j=0}^{s} b_{l-s+j} \leq z-R_{i-1},\right. \\
K_{l-s m+1}= & \left\{\left(b_{l-s}, b_{l-s+1}, \ldots, b_{l}\right) \mid \sum_{j=0}^{s} b_{l-s+j} \leq z-R_{m}, b_{l-s+n} \geq 0(0 \leq n \leq s)\right\} .
\end{aligned}
$$


Then

$$
K_{l-s}=K_{l-s p} \cup K_{l-s p+1} \cup \cdots \cup K_{l-s m} \cup K_{l-s m+1} .
$$

Put

$$
\begin{aligned}
g(\boldsymbol{b}) & =g\left(b_{l-s}, b_{l-s+1}, \ldots, b_{l}\right) \\
& =\left(H^{\prime}\left(z-b_{l}-b_{l-1}-\cdots-b_{l-s}\right)-\alpha c\right) \phi\left(b_{l-s}\right) \phi\left(b_{l-s+1}\right) \cdots \phi\left(b_{l}\right) \\
g_{t}(\boldsymbol{b}) & =g_{t}\left(b_{l-s}, b_{l-s+1}, \ldots, b_{l}\right) \\
& =\left(H_{t}^{\prime}\left(z-b_{l}-b_{l-1}-\cdots-b_{l-s}\right)-\alpha c\right) \phi\left(b_{l-s}\right) \phi\left(b_{l-s+1}\right) \cdots \phi\left(b_{l}\right) \\
& \quad(p \leq t \leq m+1),
\end{aligned}
$$

where we make a minor change when $s=l-1$. It is shown that

$$
\begin{aligned}
h_{l-s}(z) & =\iint \cdots \int_{K_{l-s}} g(\boldsymbol{b}) d b_{l-s} d b_{l-s+1} \cdots d b_{l} \\
& =\sum_{t=p}^{m+1} \iint \cdots \int_{K_{l-s t}} g(\boldsymbol{b}) d b_{l-s} d b_{l-s+1} \cdots d b_{l} \\
& =\sum_{t=p}^{m+1} \iint \cdots \int_{K_{l-s t}} g_{t}(\boldsymbol{b}) d b_{l-s} d b_{l-s+1} \cdots d b_{l}
\end{aligned}
$$

This yields us to express the function $F_{l}^{\prime}(z)$ as follow

$$
F_{l}^{\prime}(z)=H_{m+1}^{\prime}(z)-\alpha c+\sum_{i=0}^{l-1} \alpha^{i+1} h_{l-i}(z) .
$$

We need to keep on searching $\bar{x}_{l+2}, \bar{x}_{l+3}, \ldots, \bar{x}_{N}$, and so it is necessary to write $F_{l+1}^{\prime}(z), F_{l+2}^{\prime}(z), \ldots, F_{N-1}^{\prime}(z)$. Unfortunately they demand more calucations than that of $F_{l}^{\prime}(z)$ if we would search them in the general form.

We have discussed how to find $x_{N}$. However it is too difficult to deal with this inventory system even when it is rather easier case. We should develop the thoery to handle our dynamic inventory problem.

\section{References}

Kabak, I.W. (1984). Partial Returns in the Single Period Inventory Model, IE News, 19, 1-3.

Kodama, M. (1986). Probabilistic Scheduling-Period-Order-Level Models with General Demand Pattern (in Japanese), Society of Political Economy, 51, 35-44.

Kodama, M. (1990a). Probabilistic Inventory Models with Partial Returns and Additional Orders (I) (in Japanese), Society of Political Economy, 55, 31-48.

Kodama, M. (1990b). Multi-Stage Inventory Models with Various Partial Returns and Additional Orders (in Japanese), Society of Political Economy, 56, 1-26.

Kodama, M. (1991a). Probabilistic Inventory Models with Partial Returns and Additional Orders (II) (in Japanese), Society of Political Economy, 56, 277-293 
Kodama, M. (1991b). Optimal Policy for a Non-convex Expected Loss Functions(II) (in Japanese), Society of Political Economy, 57, 175-198.

Kodama, M. (1992). Optimal Policy for a Probabilistic System (I) (in Japanese), Society of Political Economy, 58, 35-50.

Kodama, M. (1993). Optimal Policy for a Probabilistic System (II) (in Japanese), Society of Political Economy, 59, 17-27.

Kodama, M. (1996). Some Probabilistic Inventory Problems with Various Demmand Pattern, Journal of Information 8 Optimization Science, 17, 17-48.

Kodama, M. (1998a). Dynamic Inventory Models with Piecewise Cost Function (I) (in Japanese), Journal of Economic Sciences, 1, 99-122.

Kodama, M. (1998b). Dynamic Inventory Models with Piecewise Cost Function (II) (in Japanese), Journal of Economic Sciences, 2, 33-60.

Kodama, M. and Kitahara, T. (1983). A Study of Unified Inventory Model on Various Demand (in Japanese), Society of Political Economy, 47, 49-72.

Kodama, M. and Sakaguchi, M. (2001a). Some comments on the probabilistic dynamic inventory problems with piecewise cost functions, Recent Developments in Oprational Reseach, Narosa New Delhi, 191-196.

Kodama, M. and Sakaguchi, M. (2001b). Probabilistic analysis of dynamic invetory models with general demand pattern, Journal of Information \& Optimization Sciences, 22, 57-72.

Kodama, M. and Sakaguchi, M. (2001c). Dynamic programming on the probabilistic inventory models with multiple piecewise cost functions, Journal of Information $\mathcal{B}$ Optimization Sciences, 22, 419-440.

Sorai, M., Arizono, I. and Ohta, H. (1986). A Solution of Single Period Inventory Model with Partial Returns and Additional Orders (in Japanese), JIMA, 37, 100-105.

Received August 3, 2001

Revised February 28, 2002

Re-revised March 26, 2002 